Check for updates

UK Health Alliance on Climate Change Cite this as: BMJ 2021;375:n2905 http://dx.doi.org/10.1136/bmj.n2905 Published: 24 November 2021

\title{
A strategy for NHS Scotland focused on reducing emissions and enhancing wellbeing
}

Elaine Mulcahy director

In 2019, the Scottish Government announced its ambition for NHS Scotland to become net-zero by 2045. After much anticipation in the lead up to COP26 in Glasgow, the NHS Scotland Climate Emergency and Sustainability Strategy was published on 10 November 2021 and opened for consultation until February 2022. ${ }^{1}$

The strategy raises the bar on the initial target by bringing forward the date for achieving net-zero emissions to 2040. Fundamental to the goal of reducing emissions is an ambition to do this is in a way that enhances wellbeing and reduces health inequalities: "A health service that improves the opportunities, life chances, health and wellbeing of every citizen in our country and fully contributes to a more cohesive, resilient and net-zero society in a just way that contributes of population wellbeing and a reduction in health inequalities."

Achieving such an ambitious aim will require commitment across all elements of the health service, including the people it serves. Significant progress in reducing emissions will be made through a shift from fossil fuels to renewables, and improving infrastructure and facilities to make them more energy efficient. The expectations of the healthcare workforce, patients, and visitors of the way in which the service operates will also need to adjust to enable the transformation to more sustainable practices, new modes of consultation, and in how they travel to and from their hospitals.

Delivery of the strategy is focused on improving sustainability across the five priority areas of: buildings and land, travel, goods and services, care, and communities.

NHS Scotland currently has 1,250 buildings as part of its estate. A commitment to invest £1o billion over 10 years to renew, reshape, and replace buildings is proposed through a programme focused on creating more resilient and sustainable healthcare facilities that use climate-considerate construction practices, have lower dependence on fossil fuels, and deliver healthier accessible internal and external environments. The impact of this work is intended to enable NHS Scotland to reduce greenhouse gas emissions from its buildings by $90 \%$ by 2040 , compared to a 1990 baseline. Significant progress on this front has already been made-greenhouse gas emissions associated with building energy use has reportedly already been reduced by $63.9 \%$, primarily as a consequence of switching from oil and coal to gas for heating, the decarbonisation of the electricity grid, and energy efficiency actions that have been taken at individual health boards.
Domestic transport is a huge contributor to Scotland's total carbon footprint, contributing an estimated $25 \%$ of the total emissions. A high dependence on motor vehicles is the primary reason for this, with data suggesting that more than half of all car journeys taken in 2019 were under $5 \mathrm{~km}-17 \%$ of them were for journeys under $1 \mathrm{~km}$. A societal and cultural shift will be needed across multiple sectors to reduce our reliance on cars and move towards cleaner modes of transport-but the systems and accessibility to do this will be vital to making any progress. NHS Scotland plans to play its part in this by changing how people access the health services they need. The strategy outlines an ambition to make it easier to walk, cycle, and access public transport to NHS services, along with plans to deliver more localised community-based facilities and reducing the number of appointments individual patients need by better integration of care services. Decarbonising the NHS fleet will also play a role in reducing the carbon footprint linked to travel. The Greener NHS England programme revealed its first electric ambulance during $\mathrm{COP}_{26} 6$ and there is hope NHS Scotland will soon be able to do the same. ${ }^{2}$

The production, supply chain, consumption, and waste linked to products and resources place a significant burden on global emissions. A shift to embracing a circular economy with a greater focus on reducing demand, reuse, and repair is outlined in the strategy as the direction in which NHS Scotland aims to move forward. About £2.5 billion is currently spent annually on goods and services purchased by the Scottish health service from as many as 8,000 different suppliers. It is impossible to measure the scale of the carbon footprint created through the manufacture, transport, delivery, use, and disposal of these products, but the opportunity to be selective in the suppliers NHS Scotland works with, the durability and recyclability of products, and their life span can all be considered in their aims to reduce emissions and waste. Another announcement made by the Greener NHS England programme in November was a commitment from its top eight suppliers to deliver their own net zero programmes over the next two decades. ${ }^{3}$ Many of the suppliers used by NHS England will also work across NHS Scotland, and there is an opportunity for Scotland to benefit from delivery of these commitments.

Central to the ambition of sustainable care is a focus on re-orienting the health service to move away from one that acts as a "national illness management service" to one that is a "national health providing service," which realises the benefits of "green health" prescribing. Four Green Health Partnerships are being piloted across Scotland to develop this approach to using nature based solutions to deliver health 
outcomes. A renewed focus on Realistic Medicine that delivers the combined benefit of potentially easing the pressures on the health working while also moving towards more sustainable practices is also outlined in the strategy. Minimising prescribing, use of medicines, and the establishment of a national green theatre programme are other examples of ways in which the delivery of sustainable care will be achieved.

The final priority outlined in the strategy focus on building sustainable communities by delivering initiatives that support health and wellbeing, develop resilience to climate change impacts, and engage with people in decision making.

There is clearly great scope across the service to adapt the way care is delivered to bring down emissions. Putting in place a strategic plan for delivery with realistic targets and annual reporting will be key to achieving its success. Also key will be ensuring the strategy becomes embedded across the service through its governance, structure, and systems. Developing and delivering new approaches for sustainable ways of working is no longer an add-on-it needs to be core to the way in which the service operates at every level.

Competing interests: Elaine Mulcahy is Head of Corporate Communications and Marketing at Royal College of Physicians and Surgeons of Glasgow.

Provenance and peer review: not commissioned, not peer reviewed

$1 \quad$ NHS Scotland climate emergency and sustainability strategy 2022 to 2026 - draft: consultation https://www.gov.scot/publications/nhs-scotland-draft-climate-emergency-sustainability-strategy

2 Zero emission ambulances show the NHS is in the driving seat in the race to net zero

https:/www.england.nhs.uk/greenernhs/2021/10/zero-emission-ambulances-show-the-nhs-isin-the-driving-seat-in-the-race-to-net-zero/

3 Healthier future inspires major NHS suppliers to step-up net zero ambitions https://www.england.nhs.uk/blog/healthier-future-inspires-major-nhs-suppliers-to-step-up-net-zero-ambitions/ 